\title{
ENFOQUE SINDRÓMICO PARA EL DIAGNÓSTICO Y MANEJO DE ENFERMEDADES INFECCIOSAS FEBRILES AGUDAS EN SITUACIONES DE EMERGENCIA
}

\author{
César Cabezas ${ }^{1,2, a}$, Fernando Donaires ${ }^{1,2, a}$
}

\begin{abstract}
RESUMEN
Varias de las enfermedades agudas con etiología infecciosa (viral, bacteriana o parasitaria) cursan inicialmente con un síndrome que puede ser febril, febril ictérico, febril hemorrágico, febril con manifestaciones respiratorias, febril con compromisio neurológico, febril con erupciones dérmicas, febril anémico, o pueden ocurrir muertes de pacientes febriles sin un diagnóstico definido, con algunas variantes según el nicho ecológico-social en el que se dan; es importante el enfoque sindrómico, para luego tipificar el caso o el brote y así tener un diagnóstico adecuado y oportuno que permita orientar tanto el manejo individual como las medidas de prevención y control en la población, aun cuando haya limitaciones para el diagnóstico de todos los casos. En este artículo se describe el enfoque sindrómico, los síndromes de importancia en salud pública (colectiva) y su abordaje, todo ello es importante por su presentación en brotes que vienen ocurriendo en los variados nichos ecológicos de nuestro país, exacerbados por el cambio climático.
\end{abstract}

Palabras clave: Infecciones por Arbovirus, dengue, Virus Zika, leptospirosis (fuente: DeCS BIREME).

\section{SYNDROMIC APPROACH FOR THE DIAGNOSIS AND MANAGEMENT OF ACUTE FEBRILE INFECTIOUS DISEASES IN EMERGENCIES}

\begin{abstract}
Several of the acute diseases with infectious etiology (viral, bacterial or parasitic) initially start with a syndrome which can be febrile, febrile icteric, febrile hemorrhagic, febrile with respiratory manifestations, febrile with neurological impairment, febrile with dermal eruptions, febrile anemic, diarrheal, necrotic cutaneous ulcer, or deaths of febrile patients may occur without a defined diagnosis, with some variants according to the ecological-social niche in which they occur; the syndromic approach is important, to quickly classify the case or the outbreak and thus have an appropriate and timely diagnosis that allows to orient both the individual management and the prevention and control measures in the population, even if there are limitations for the diagnosis of all the cases. This article describes the syndromic approach, the syndromes of importance in public health (collective) and their approach, all this is important because of its presentation in outbreaks that are occurring in the various ecological niches of our country, exacerbated by climate change.
\end{abstract}

Key words: Arbovirus Infections, dengue, Zika Virus leptospirosis (source: MeSH NLM).

\section{INTRODUCCIÓN}

Varias de las enfermedades con etiología infecciosa se presentan, desde el punto de vista clínico, con un síndrome, el que puede ser febril, febril ictérico, febril hemorrágico, febril con manifestaciones respiratorias, o febril con compromisio neurológico, particularmente en los trópicos, con algunas variantes según el nicho ecológico-social en el que se dan. Existe un perfil etiológico que es prevalente en cada nicho ecológicosocial, debiendo considerarse las etiologias parasitarias (malaria), bacterianas (leptospirosis, bartonelosis, peste), virales (dengue, chikungunya, zika, mayaro, oropouche, encefalitis equina venezolana, hantavirus, fiebre amarilla, etc), riketsiosis (tifus murino, tifus exantemático), entre los más frecuentes en nuestro país (Figura 1) ${ }^{(1,2,3)}$.

\footnotetext{
Instituto Nacional de Salud. Lima, Perú.

Facultad de Medicina, Universidad Nacional Mayor de San Marcos. Lima, Perú.

Médico infectólogo.

Recibido: 19/04/2017 Aprobado: 07/06/2017 En línea: 28/06/2017
}

Citar como: Citar como: Cabezas C, Donaires F. Enfoque sindrómico para el diagnóstico y manejo de enfermedades infecciosas febriles agudas en situaciones de emergencia. Rev Peru Med Exp Salud Publica. 2017;34(2):316-22. doi: 10.17843/rpmesp.2017.342.2836 


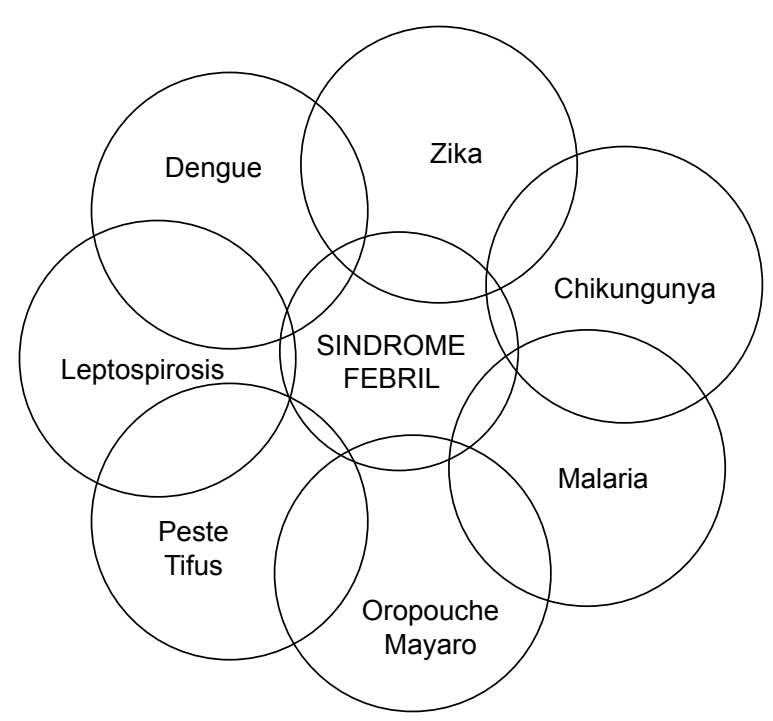

Figura 1. Etiologías que deben considerarse en el síndrome febril, según frecuencia en los diferentes nichos ecológicos en el Perú

Existen áreas endémicas de malaria en el Perú, particularmente en la Amazonía y en la costa norte, aunque actualmente está circunscrita a la Amazonía ${ }^{(4)}$. El dengue está disperso en el país, econtrándose, prácticamente, el todas las ciudades amazónicas y en la costa desde Tumbes hasta Ica, favorecido en la Amazonía por la lluvias y en la costa, principalmente, por la falta de acceso al agua, lo que obliga a los pobladores a almacenarla de manera inadecuada y se transforma en un factor de riesgo para tener criaderos de Aedes aegyti ${ }^{(5,6)}$, últimamente, debido al fenómeno El Niño Costero y el cambio climático en general, se hace más dificil el control de este vector.

La fiebre por chikungunya ha emergido en las Américas y, desde fines del año 2014, viene afectando al norte de país; el vector de este virus es también el zancudo Aedes aegypti, y la dispersión de la infección por el país es inminente ${ }^{(7)}$. El virus Zika, que cursa también con fiebre y que se inició en el Brasil $(7,8)$, se ha dispersado por las Américas, incluyendo el Perú, (Jaén, lquitos, Chincha) ${ }^{(9)}$; se ha demostrado la asociación de la infección por el virus Zika y la microcefalia; asimismo, se ha descrito un síndrome congénito con compromiso neurológico en recién nacidos ${ }^{(7)}$, de gran impacto en la salud de estos niños y en la economía de las familias y del estado por el costo de la rehabilitación. El riesgo de dispersión es inminente, si tenemos en cuenta las migraciones y que tiene como vector común al mismo Aedes aegypti ${ }^{(10)}$.

Una enfermedad endémica, particularmente en la selva alta del Perú, es la fiebre amarilla ${ }^{(11)}$ que se da en población migrante no inmunizada que se desplaza a la selva, pero tambien en población que vive en esta zona endémicas y no está vacunada. En Brasil y en Africa se vienen dando epidemias de fiebre amarilla de grandes proporciones con alta letalidad ${ }^{(12)}$.
La leptospirosis, en los últimos años, se ha convertido en un serio problema, particularmente en la Amazonía. Actualmente, como consecuencia de las abundantes lluvias y en el contexto del fenómeno El Niño Costero, se vienen presentando brotes de dengue, chikungunya y leptospirosis en la costa peruana, principalmente en el norte y centro de esta región geográfica ${ }^{(9)}$. Otro agente que cursa con fiebre y está presente en la Amazonía es la riketsiosis ${ }^{(13)}$, pero también se han descrito casos de síndrome febril debido al tifus murino (Rickettsia typhi) en áreas de la sierra de Piura luego del fenómeno $\mathrm{El}$ Niño en el año 2008. La infección por hantavirus es otro problema emergente en la Amazonía, que también cursa con síndrome febril para luego tener complicaciones respiratorias ${ }^{(14)}$ y la exposición de la población a excretas de roedores silvestres luego de los huaicos (Iloqllas) siempre son un riesgo. En los establecimientos de salud de atención primaria en estas áreas, los pacientes no vienen con un diagnóstico establecido, sino con signos y síntomas que constituyen síndromes, siendo el más frecuente el sindrome febril, sin un foco aparente de infección que explique la fiebre; por lo que es importante enfrentar los problemas de salud con lo que vienen los pacientes, primero delimitando la etiología causante del síndrome en los conglomedados y su consiguiente manejo clínico-terapéutico. Adicionalmente, muchas veces los primeros casos que se ven pueden corresponder al inicio de brotes en las poblaciones, por lo que se debe pensar, además del manejo individual del caso, en la posibilidad de un brote epidémico y dar la alerta para su control y prevención, lo cual implica la participación de otros grupos de trabajo, como los de control del medioambiente y de control de vectores para hacerlo efectivo en sus fases larvaria y adulta.

Basados en estudios previos, se puede contar con la etiología más frecuente en determinados nichos ecológicos; según esta información, se seguirá un algoritmo para el diagnóstico y el manejo, si corresponde a malaria, dengue, zika, mayaro, oropouche, EEV, leptospirosis, hantavirus, fiebre amarilla, o riketsiosis ${ }^{(15)}$.

En este contexto, es necesario precisar algunas definiciones antes de proponer el diagnóstico con un enfoque sindrómico:

Síndrome: es el conjunto de síntomas y signos que pueden tener una fisiopatología común, pero que responden a diferentes etiologías.

Fiebre -para fines prácticos- es la elevación de la temperatura axilar a un valor mayor o igual a $38^{\circ} \mathrm{C}$.

Enfermedad aguda: cuando su evolución tenga menos de 7 días de duración desde el inicio de los síntomas.

Conglomerado: es una agrupación de casos de un síndrome en particular, en un lugar o en un periodo determinado, que se considera mayor de lo usual o esperado. 
Gravedad de una enfermedad: cuando el paciente requiere hospitalización o tiene alguna de estas manifestaciones: colapso circulatorio, insuficiencia orgánica grave, pérdida de conciencia o el paciente fallece.

Los signos de gravedad y signos de alerta, así como los factores de riesgo que pueden agravar la condición de un paciente, se pueden ver en la Tabla 1.

\section{SÍNDROMES DE IMPORTANCIA EN LA SALUD PÚBLICA}

Síndrome febril. Todo paciente o conglomerado de pacientes con inicio brusco de fiebre sin foco infeccioso aparente de menos de 7 días de evolución. Las causas pueden ser parasitarias (malaria, enfermedad de Chagas), bacterianas (leptospirosis, bartonelosis, peste), virales (dengue, chikungunya, zika, mayaro, oropouche, encefalitis equina venezolana, hantavirus, etc), riketsiosis (tifus murino, tifus exantemático), entre los más frecuentes en nuestro medio.

Síndrome febril ictérico agudo. Todo paciente o conglomerado con presentación brusca de fiebre, ictericia y ausencia de factores predisponentes conocidos en el paciente (p.e. hepatopatía crónica, hepatopatía inducida por fármacos y hepatopatías autoinmunes). Las causas pueden ser hepatitis virales A, B/Delta, C, E, fiebre amarilla, leptospirosis, bartonelosis (enfermedad de Carrión).

En la Figura 2 se puede apreciar comparativamente las principales manifestaciones clínicas de infecciones por arbovirus que actualmente cocirculan en varias regiones del país como el dengue, zika y chikungunya ${ }^{(16)}$. También hay que considerar en el diagnóstico diferencial de la leptospirosis, la malaria, entre otras enfermedades que cursan con fiebre y que al inicio son difíciles de diferenciarlas clínicamente.

Síndrome febril respiratorio agudo. Todo paciente o coglomerado mayor de 5 años de edad con inicio brusco de fiebre, acompañado de tos o dificultad respiratoria y ausencia de factores predisponentes conocidos en el paciente. Las etiologías pueden corresponder a influenza, peste neumónica, leptospirosis, ántrax neumónico, hanta, SARS entre otros.

Síndrome febril con manifestaciones neurológicas. Todo paciente o conglomerados de febriles, con presentación aguda de alteración del sistema neurológico, definida por la presencia de cualquiera de los siguiente: deterioro agudo de la función mental, por ejemplo:

- Pérdida de la memoria, comportamiento anormal, alteración de la conciencia).

- Aparición aguda de parálisis.

- Convulsiones.

- Signos meníngeos.

- Movimientos involuntarios (por ejemplo: corea, temblor, mioclonus).

- Otro síntoma grave que se crea que es una disfunción del sistema neurológico.

Lo anterior en ausencia de factores predisponentes conocidos en el paciente, como: alteraciones metabólicas, insuficiencia renal crónica, hepatopatía crónica, diabetes mellitus, tirotoxicosis, etc.

Síndrome febril con erupción dérmica. Todo paciente o conglomerado, caracterizado por cuadro febril de menos de 7 días de duración acompañado de erupción dérmica (exantemática, papular o vesicular). Las etiologías pueden corresponder a: sarampión, zika, dengue, rubéola, tifus o varicela.

Tabla 1. Signos de gravedad y signos de alerta en la evolución de un síndrome o enfermedad determinada

\begin{tabular}{ll}
\hline Signos de gravedad & \multicolumn{1}{c}{ Signos de alerta } \\
\hline - Presión sistólica $<80 \mathrm{mmHg}$ & - Disnea, tos o taquipnea \\
- Presión sistólica $<90 \mathrm{mmHg}$ refractaria a volumen & - Oliguria o anuria \\
administrado & - Vómitos frecuentes \\
- Frecuencia cardiaca $<40$ latidos por minuto o $>150$ & - Dolor abdominal \\
$\begin{array}{l}\text { latidos por minuto. } \\
\text { - Arritmias o taponamiento cardíaco }\end{array}$ & - Sangrado de mucosas \\
$\begin{array}{l}\text { - Insuficiencia respiratoria aguda } \\
\text { - Hemorragias graves }\end{array}$ & - Necesidad de aislamiento \\
- Coma o crisis convulsivas & Factores de riesgo o comorbilidades \\
& - Gestantes, mayores de 65 años y niños pequeños \\
& - Comorbilidades: diabetes mellitus, hipertensión arterial, artritis \\
& reumatoide, VIH/SIDA, neoplasias. \\
\hline
\end{tabular}




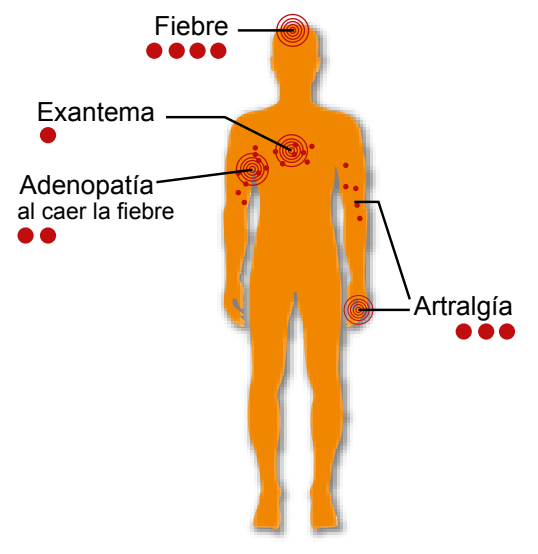

Dengue

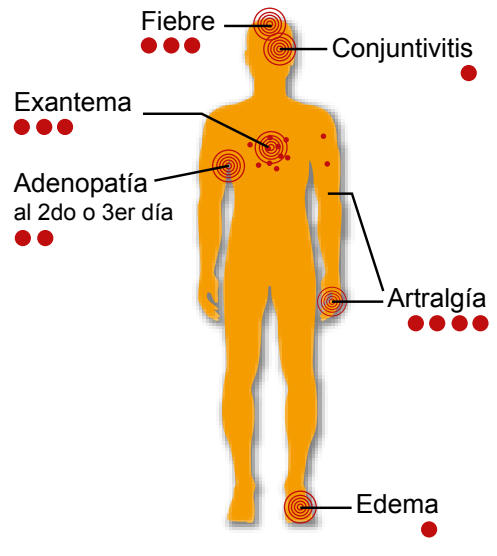

Chikungunya

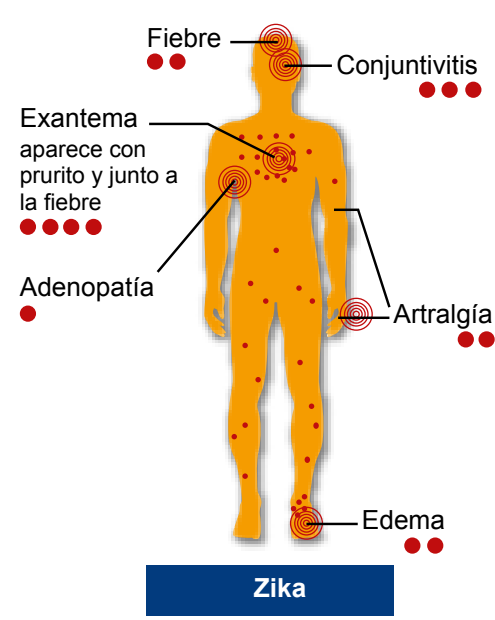

Figura 2. Signos y síntomas más destacados en el dengue, chikungunya y zika

Síndrome febril anémico agudo. Paciente o conglomerado de pacientes que presenta fiebre $y$ palidez marcada de piel o mucosas o hematocrito $<30 \%$, como puede ocurrir en la enfermedad de Carrión, la malaria por Plasmodium falciparum

La vigilancia sindrómica, -que algunos países han optado por utilizarla- está orientada a detectar rápidamente la presencia de brotes con potencial daño a la salud pública, considerando no solo los brotes de origen infeccioso conocidos, sino también los de origen desconocido. Este tipo de vigilancia tiene algunas ventajas como que son:

- Definiciones de casos simples y estables

- Se refieren a hallazgos clínicos.

- Es de notificación fácil.

- Capta gran volumen de datos y puede detectar enfermedades desconocidas para la zona.

- Reducción de la sobrecarga sobre los servicios de salud (notificación previa al diagnóstico, asistencia rápida, reducción de formatos, etc.).

En el contexto descrito previamente, en particular en el inicio de enfermedades infecciosas, es importante el enfoque sindrómico, y como se puede ver en la figura 3, el proceso para el diagnóstico y el manejo empieza con identificar el problema con el que viene el paciente, recabar de la mejor forma posible la información epidemiológica como los antecedentes de exposición a riesgos, a otros pacientes, así mismo, información clínica a través de una exhaustiva anamnesis y examen clínico en busca de un foco infeccioso. Con esta información se constituye un síndrome - como los previamente descritos-. Con toda esta información se orientará racionalmente la indicación de una prueba diagnóstica de laboratorio u otro soporte que apoye el diagnóstico como el de imágenes.

Definido el diagnóstico etiológico, se podrá orientar mejor las medidas terapéuticas, de soporte y de control. Es importante considerar las medidas a nivel individual de la persona afectada, como también al nivel colectivo, dada la potencialidad del desarrollo de epidemias, por lo que es imprescindible la notificación a las autoridades sanitarias y tomar las medidas de prevención-control en la comunidad.

Para la confirmación de los casos es necesario el uso de pruebas de laboratorio, sobre todo cuando hay que tipificar un brote - idealmente en su inicio-, para luego utilizar el nexo epidemiológico como un criterio para enfrentar los casos que tiene características similares a los primeros diagnosticados. En la Figura 4 se describen las principales pruebas utilizadas para este fin. Es importante tener en cuenta el tiempo de enfermedad al momento de la obtención de la muestra, porque este dato nos permitirá solicitar la prueba más adecuada. Así en las infecciones por arbovirus (dengue, zika, chikungunya, fiebre amarilla) en los primeros cinco días -en promedio- se da el periodo de viremia, por lo que las pruebas indicadas serían el PCR en tiempo real, la determinación de antígenos virales (NS1 en el dengue) mediante ELISA o pruebas rápidas y el aislamiento viral. Los primeros son de mayor uso para fines de diagnóstico más oportuno, aunque siempre hay que 


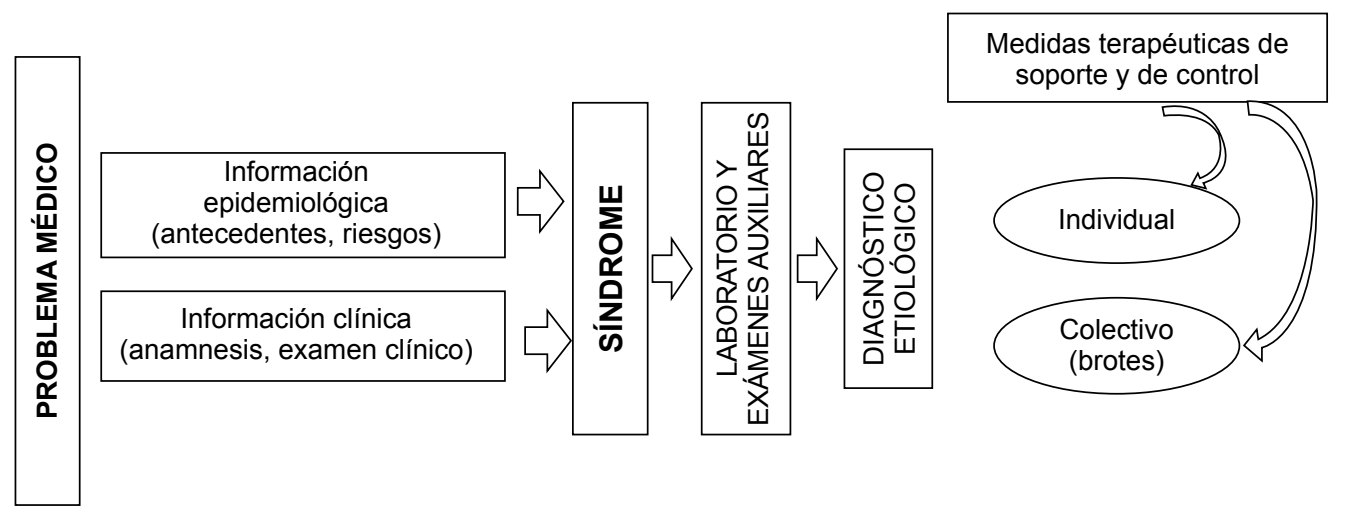

Figura 3. El proceso del diagnóstico, bajo el enfoque sindrómico

considerar las limitaciones que puedan tener en cuanto a sensibilidad y especificidad. En el caso del Zika se puede encontrar el virus por la técnica del PCR en la orina hasta las 2 semanas, y en semen hasta los seis meses cuando sea necesario realizar.

Después de los cinco días - para diagnóstico de arbovirus- ya no existe viremia, pero aparecen los anticuerpos IgM, los cuales se pueden determinar mediante la prueba de ELISA. Para el caso de la leptospirosis, la malaria y la peste, las pruebas a ser utilizadas al momento de captar al paciente febril, como se indican en la figura 4.

\section{DIAGNOSTICO Y MANEJO EN EL CONTEXTO DE BROTES}

En situaciones de brotes, mediante el enfoque sindrómico se orienta las pruebas de laboratorio para inicialmente confirmar y tipificar el brote, y así luego actuar (a nivel individual y colectivo) asumiendo que los casos corresponden a la etiología determinada en los casos estudiados.

Muchas veces es imposible que todos los casos tengan un diagnóstico de laboratorio, por lo que una vez tipificado el brote se asume el diagnóstico por nexo epidemiológico.

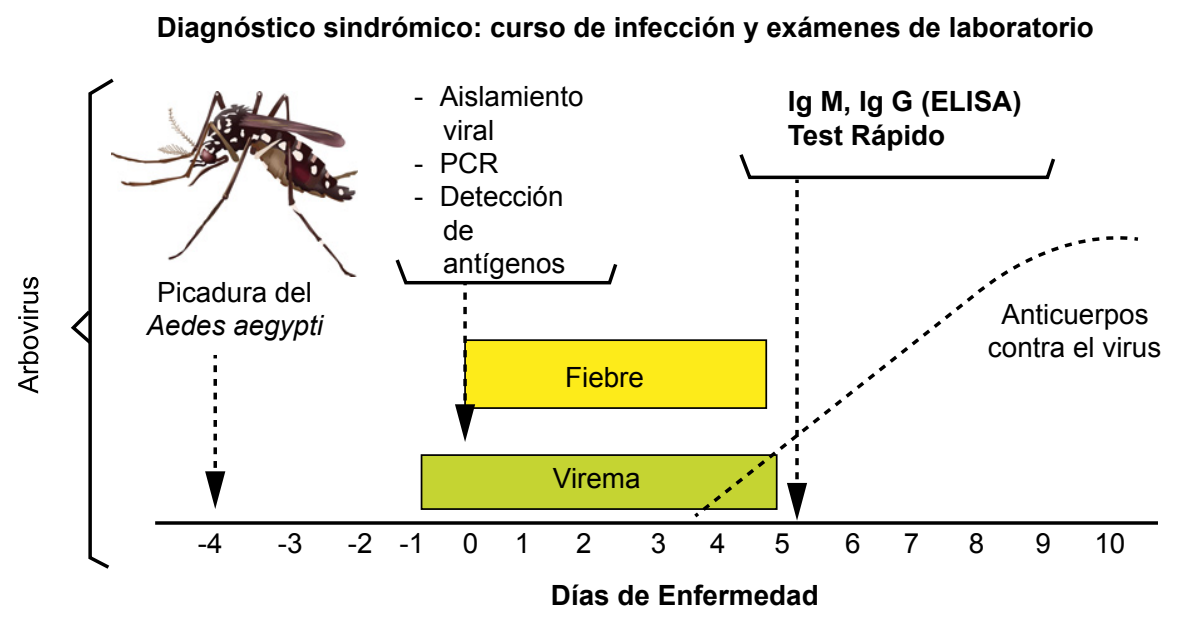

Leptospira: PCR, ELISA IgM, MAT, pruebas rápidas IgM/lgG al momento de captar el paciente

Malaria: Gota gruesa, pruebas rápidas al momento de captar el paciente

Peste: Examen directo de aspirado de bubón, PCR, prueba rápida (anticuerpos anti F1)

Figura 4. Diagnóstico sindrómico: curso de la infección y exámenes de laboratorio 
En los pacientes que han requerido hospitalización, es importante contar con un diagnóstico etiológico, en lo posible, para lograr un manejo y seguimiento adecuados.

En desastres y en brotes casi siempre hay limitaciones para el diagnóstico individual de laboratorio, aunque es necesario catalogar la etiología en el conglomerado que se está evaluando, y el objetivo es limitar la mortalidad, por lo que el manejo clínico es vital. Así, si estamos frente a un caso de síndrome febril agudo, en zonas donde cocirculan arbovirus como el dengue y leptospirosis, hay que avaluar los signos de alarma, signos de gravedad o si pertenece a un grupo de riesgo. Si no presenta estas manifestaciones y está estable, iniciar tratamiento por vía oral para leptospirosis (amoxicilina o azitromicina -en alérgicos al primero) y brindar el manejo ambulatorio del dengue (criterio de grupo $\mathrm{A}$ del manejo de dengue). De lo contrario, si hay signos de alarma, gravedad o el paciente pertenece a uno de los grupos de riesgo ${ }^{(16)}$ se debe iniciar con el tratamiento antibiótico parenteral (ceftriaxona) y manejo con fluidos (criterio de grupo B-C de manejo de dengue). En este punto es importante indicar que el monitoreo no invasivo de la presión arterial media (PAM) y frecuencia cardiaca (FC) debe ser estricta durante el periodo crítico del dengue, donde se presenta la extravasación y el objetivo del tratamiento es mantener la volemia, evitando ir a la sobrehidratación (https://www.youtube.com/channel/ UCgwb5fMMucCDtshi4vYASiw/videos).

Dado nuestro complejo territorio y por el acceso limitado a los servicios de salud, hay que remarcar la importancia de primero estabilizar al paciente hemodinámicamente, antes de derivarlo a un establecimiento de mayor complejidad. Para dar el tratamiento presuntivo, debe contarse con información previa del patógeno que está circulando en determinado nicho ecológico, así, en la costa norte ocurre la cocirculación de arbovirus y leptospiras, en la Amazonía puede darse la cocirculación de arbovirus, leptospira y malaria. Si bien no es factible tener el diagnóstico de todos los casos en una epidemia, es importante saber los agentes que circulan para tomar las medidas más apropiadas tanto a nivel individual como colectivo.

Finalmente, la importancia del enfoque sindrómico, radica en que permite una rápida aproximación con casos que pueden estar relacionadas a epidemias con gran potencial de diseminación, transmisión rápida; letalidad inesperadamente alta, inexistencia de tratamiento eficaz o resistencia a drogas antimicrobianas disponibles.

La mayor frecuencia de eventos debidos a los cambios climáticos hace necesario recurrir al enfoque sindrómico. También creemos conveniente el desarrollo y la adecuación de las tecnologías que respondan a problemas sanitarios como los que afrontamos, permitiendo un diagnóstico oportuno al inicio de los brotes e igualmente tengamos la organización y adecuación de los servicios de salud. Evidentemente lo mejor será el desarrollo de a acciones preventivas de estas enfermedades o el control rápido cuando sea inevitable su prevención.

Fuentes de financiamiento: autofinanciado.

Conflictos de interés: los autores manifiestan no tener conflictos de interés en la publicación del artículo.

Contribuciones de autoría: CC y FD contribuyeron con la concepción y diseño del artículo, revisión bibliográfica, redacción del artículo, revisión crítica del artículo, y aprobación de la versión final.

\section{REFERENCIAS BIBLIOGRÁFICAS}

1. Halsey ES, Siles C, Guevara C, Vilcarromero S, Jhonston EJ, Ramal C, et al. Mayaro virus infection, Amazon Basin region, Peru, 2010-2013. Emerg Infect Dis. 2013;19(11):1839-42.

2- Forshey BM, Guevara C, LagunaTorres VA, Cespedes M, Vargas J, Gianella A et al. (2010) Arboviral Etiologies of Acute Febrile Illnesses in Western South America,

2000-2007. PLoS Negl Trop Dis 4(8): e787. doi:10.1371/journal. pntd.0000787

3.. Watts DM, Phillips I, Callahan JD, Griebenow W, Hyams KC, Hayes CG. Oropouche virus transmission in the Amazon River basin of Peru. Am J Trop Med Hyg. febrero de 1997;56(2):148-52.
4. Griffing SM, Gamboa D, Udhayakumar $\mathrm{V}$. The history of 20th century malaria control in Peru. Malar J. 2013;12:303.

5. Cabezas C, Fiestas V, García-Mendoza M, Palomino M, Mamani E, Donaires F. Dengue en el Perú: a un cuarto de siglo de su reemergencia. Rev Peru Med Exp Salud Pública. 2 de abril de 2015;32(1):146-56.

6. Cabezas S C. Dengue en el Perú: Aportes para su diagnóstico y control. Rev Peru Med Exp Salud Publica. julio de 2005;22(3):212-28.

7. Zanluca C, Melo VCA de, Mosimann ALP, Santos GIV dos, Santos CND dos, Luz K, et al. First report of autochthonous transmission of Zika virus in Brazil. Mem Inst Oswaldo Cruz. junio de 2015;110(4):569-72.
8. Jaenisch T, Rosenberger KD, Brito C, Brady O, Brasil P, Marques ET. Risk of microcephaly after $Z$ ika virus infection in Brazil, 2015 to 2016. Bull World Health Organ. 1 de marzo de 2017;95(3):191-8.

9. CNEPCE, MINSA. Boletín epidemiológico del Perú. Volumen 26 SE 12 [Internet]. 2017. Disponible en: http://www.dge.gob.pe/portal/docs/ vigilancia/boletines/2017/12.pdf

10. DGE, MINSA. Alerta: Primer caso autóctono de Chikungunya y Riesgo de transmisión en el Perú [Internet]. 2015. Disponible en: http://www. dge.gob.pe/portal/docs/alertas/2015/ AE005.pdf

11. Espinoza SM, Cabezas SC, Ruiz OJ. Un acercamiento al conocimiento de la fiebre amarilla en el Perú. Rev Peru 
Med Exp Salud Publica. octubre de 2005;22(4):308-15.

12. WHO Yellow fever - Brazil [Internet]. WHO. [citado 19 de abril de 2017]. Disponible en: http://www. who.int/csr/don/20-march-2017yellow-fever-brazil/en/

13. Sihuincha M M, Anaya F E, Carranza V V, Durand V S. Evidencia serológica de la presencia de Rickettsias del grupo de la fiebre manchada en la Amazonía del Perú. Rev Peru Med Exp Salud Publica. octubre de 2006;23(4):284-7.
14. García MP, Sinti P, Herrera AL, Donaires F, Álvarez C, Arrasco J, et al. Confirmación etiológica de los dos primeros casos de hantavirosis humana en el Perú. Rev Peru Med Exp Salud Pública. 30 de septiembre de 2011 [citado 19 de abril de 2017];28(3).

15. Troyes RL, Fuentes TL, Troyes RM, Canelo DL, García MMP, Anaya FE, et al. Etiología del síndrome febril agudo en la provincia de Jaén, Perú 2004-2005. Rev Peru Med Exp Salud Pública.2006; 23(1):5-11
16. OPS, OMS. Instrumento para el diagnóstico y la atención a pacientes con sospecha de arbovirosis [Internet]. OPS; 2016 [citado 19 de abril de 2017]. Disponible en: http://iris.paho. org/xmlui/handle/123456789/31448

Correspondencia: César Cabezas Sánchez Dirección: Capác Yupanqui 1400, Jesús María. Lima, Perú.

Correoelectrónico: ccabezas@ins.gob.pe

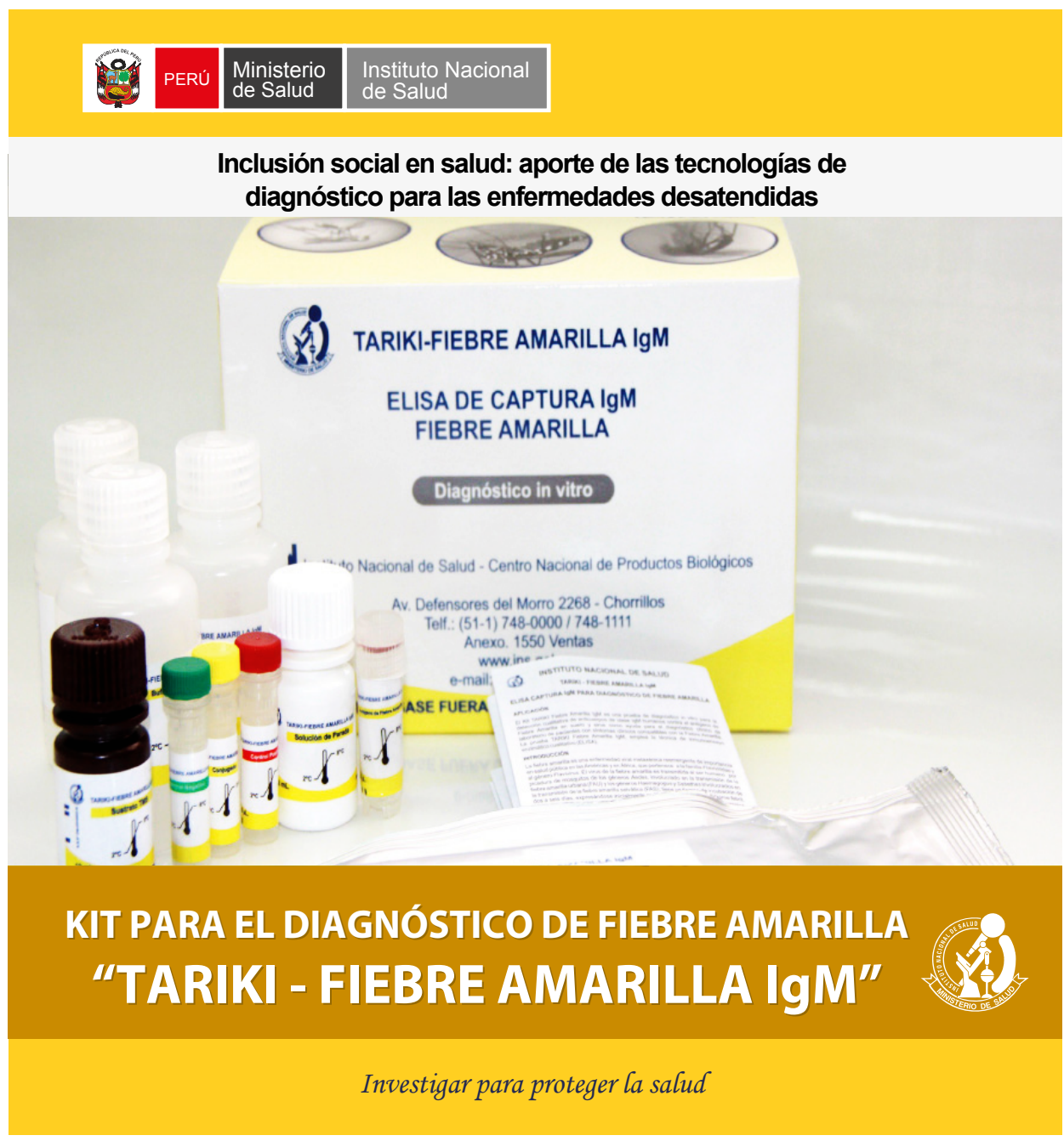

